\title{
A TRANSFORMAÇÃO DIGITAL E A GESTÃO DO CONHECIMENTO: CONTRIBUIÇÕES PARA A MELHORIA DOS PROCESSOS PRODUTIVOS E ORGANIZACIONAIS
}

\author{
Ricardo Alexandre Diogo \\ Pontifícia Universidade Católica do Paraná (PUCPR) \\ Universidade Federal de Santa Catarina (UFSC) \\ r.diogo@pucpr.br \\ Armando Kolbe Junior \\ Uninter \\ Universidade Federal de Santa Catarina (UFSC) \\ 1 armando.k@uninter.com \\ Neri dos Santos \\ Universidade Federal de Santa Catarina (UFSC) \\ nerisantos@gmail.com
}

\begin{abstract}
Resumo
A Transformação Digital (TD), também chamada de Indústria 4.0, tem implementado tecnologias para o aperfeiçoamento dos processos produtivos e de gestão organizacional. Diante desse cenário, o presente estudo pretende verificar como a Gestão do Conhecimento $(\mathrm{GC})$ pode contribuir para a TD nas organizações e, ao mesmo tempo, como a TD está colaborando para a GC. Sendo assim, primeiramente a GC é contextualizada junto aos conceitos de TD. Então, para se atingir o objetivo deste estudo, uma revisão sistemática da literatura foi realizada, considerando a relação dos pilares da Indústria 4.0 e dos Sistemas Ciber-Físicos com a GC. Para finalizar, os resultados da revisão sistemática são analisados e discutidos para responder ao objetivo deste trabalho.
\end{abstract}

Palavras-chave: Transformação Digital. Indústria 4.0. Sistemas ciber-físicos. Gestão do Conhecimento.

\section{DIGITAL TRANSFORMATION AND KNOWLEDGE MANAGEMENT: CONTRIBUTIONS TO IMPROVING PRODUCTIVE AND ORGANIZATIONAL PROCESSES}

\begin{abstract}
Digital Transformation (TD), also called Industry 4.0, has implemented technologies for the improvement of production processes and organizational management. Given this scenario, the present study intends to verify how Knowledge Management (CG) can contribute to TD in organizations and, at the same time, how TD is collaborating for CG. Therefore, the CG is first contextualized with TD concepts. Therefore, in order to achieve the objective of this study, a systematic review of the literature was carried out, considering the relationship between the pillars of Industry 4.0 and the Cyber-Physical Systems with GC. To conclude, the results of the systematic review are analyzed and discussed to answer the objective of this work.
\end{abstract}

Keywords: Digital Transformation. Industry 4.0. Cyber-physical systems. Knowledge management. 


\section{INTRODUÇÃO}

A Transformação Digital (TD), ou Indústria 4.0, como é comumente chamada, tem sido factível com a constante inclusão de tecnologias inovadoras nos diferentes setores econômicos. Esse movimento é apontado como a $4^{a}$ Revolução Industrial, que se utiliza dos sistemas ciberfísicos (CPS), para modernizar a indústria, tornando a automação holística com a integração das funções de produção e negócios além das fronteiras da organização (LYDON, 2016).

Se há evidentes transformações acontecendo nas organizações, há também mudanças na forma de gerir os negócios. Isso significa promover melhorias nos meios produtivos e de gestão, que reflete na capacitação da mão-de-obra para a ocupação de novos postos de trabalho. Portanto, há a necessidade do emprego das metodologias para a Gestão do Conhecimento (GC) aliadas ao que a TD oferece. Diante desse cenário, a seguinte questão de pesquisa surge: "Como a GC pode contribuir para a TD nas organizações e, em contrapartida, como a TD pode contribuir para implementar a GC nas organizações?”. Para responder a essa pergunta, foi realizada uma revisão sistemática, com busca de publicações na base de dados Scopus. A resposta a essa questão de pesquisa pode direcionar outros estudos, ajudando na implementação da TD e GC nos meios produtivos e nos negócios empresariais.

As próximas seções estão organizadas de forma a definir o que é a TD e os seus nove pilares, seguido pela contextualização da GC. Em seguida, o Design da Pesquisa é apresentado para mostrar como o estudo foi realizado. Na sessão de Resultados, os trabalhos são discutidos, considerando como a GC se relaciona com os 9 pilares da Indústria 4.0. Por fim, as Considerações Finais são apresentadas.

\section{DESENVOLVIMENTO}

Nesta seção, são apresentados os constructos usados no presente trabalho de pesquisa: a Transformação Digital e a Gestão do Conhecimento.

\subsection{TRANSFORMAÇÃO DIGITAL}

A Transformação Digital, termo adotado pelo governo brasileiro (BRASIL, 2017), começou a ser pensada em 2006, através de um "Plano Estratégico Alemão de Alta Tecnologia" (LYDON, 2016). Quatro anos mais tarde, esse plano se tornou a "Iniciativa Estratégica de Alta Tecnologia para 2020", com objetivos de se ter a visão integrada da indústria, alavancando os sistemas computacionais e as tecnologias para Internet. Por fim, um relatório, chamado de 
"Recomendações para Implementação da Iniciativa Estratégica Indústria 4.0", foi gerado. Dentre as recomendações do documento, estão presentes o desenvolvimento de tecnologias para Internet das Coisas (IoT), as comunicações e os serviços web para a manufatura. Além disso, o relatório aponta que as fábricas precisam se modernizar para se tornarem inteligentes, através da automação holística. E ainda, a atualização deve abranger a logística interna e externa, o setor de marketing e de serviços, o que leva a integração das funções de produção e negócios além das fronteiras das organizações.

O movimento alemão foi seguido por outros países, como a China (WUEBBEKE et al., 2016), o Japão (IVI, 2018), os Estados Unidos da América (SMART MANUFACTURING LEADERSHIP COALITION, 2012) e o Brasil (BRASIL, 2016, 2017, 2018). É uma mudança que tem sido considerada a $4^{a}$ Revolução Industrial (Figura 1), caracterizada pela presença dos Sistemas Ciber-Físicos (CPS) nos meios de produção, produtos e serviços. Os CPS são compostos de hardware e software integrados que são capazes de controlar e monitorar equipamentos físicos.

Figura 1. As 4 Revoluções Industriais

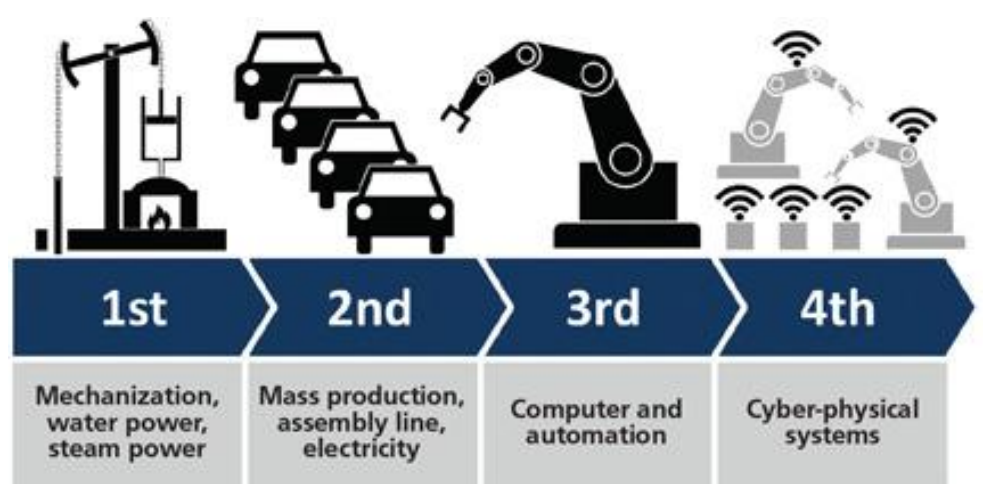

Fonte: (LYDON, 2016).

Contudo, não são apenas os CPS que marcam a $4^{\mathrm{a}}$ Revolução Industrial. A TD é suportada por nove pilares (LYDON, 2016; RÜBMANN et al., 2015), que são descritos na sequência (Figura 2).

\subsubsection{Robótica Autônoma e Colaborativa}

A robótica está presente nos meios produtivos desde a $3^{\text {a }}$ Revolução Industrial. Agora, na Indústria 4.0, há dois tipos de robôs em destaque: o colaborativo e o autônomo. O primeiro é a evolução dos tradicionais robôs industriais, sendo estes geralmente caracterizados pela presença de 6 eixos imitando os movimentos antropomórficos de um braço. Os robôs colaborativos são dotados de sensores avançados que permitem a percepção da presença 
humana em seu volume de trabalho, fazendo com que o controle reduza a velocidade dos movimentos a uma situação segura de operação. E mesmo que haja uma colisão com pessoas, há sensores de força para a completa parada do robô. Esse avanço tecnológico é que permite a operação segura dos robôs colaborativos junto aos trabalhadores em linhas de produção.

Figura 2. Os 9 pilares da Indústria 4.0

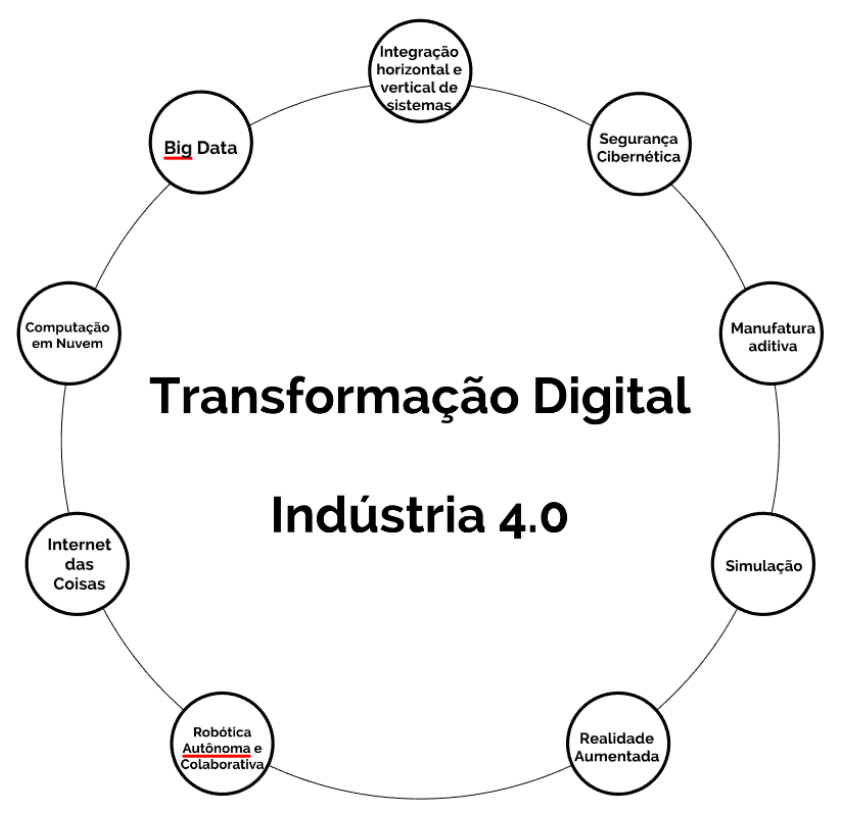

Fonte: Adaptado de (LYDON, 2016).

O segundo tipo de robô para a TD é o autônomo. Os antigos seguidores de linhas, que fazem o transporte robotizado de peças nas indústrias, e chamados de AGV (Automated Guided Vehicle), evoluíram para IGV (Intelligent Guided Vehicle). Antes, esses robôs eram controlados por uma central de operação. Os atuais possuem controle embarcado e com inteligência suficiente para se georreferenciarem em fábricas sem o uso das "linhas", além de desviarem de obstáculos nas rotas e detectarem os destinos automaticamente.

\subsubsection{Internet das Coisas (IoT)}

A IoT está se tornando presente em qualquer "coisa", o que permite conectividade de máquinas e processos industriais, produtos, serviços e pessoas. Na indústria tem se escolhido o termo IIoT (Industrial Internet of Things) ou simplesmente Internet Industrial (ABII, 2018). A conexão entre pessoas é comum através dos smartphones, mas também de pessoas com sistemas computacionais. Os wearebles permitem que informações fisiológicas sejam carregadas instantaneamente em plataformas computacionais na nuvem, fazendo com que haja conhecimento sobre o estado de saúde corporal. Outro exemplo é a cápsula endoscópica, um 
dispositivo insideable para exames laboratoriais sem a necessidade de o paciente ficar na clínica.

\subsubsection{Computação em Nuvem}

Aonde quer que os dispositivos estejam, é necessário que eles se conectem em sistemas computacionais que o usuário sequer sabe onde estão. Para que a IoT seja possível em dispositivos cada vez menores e com capacidade de memória limitada, a presença da computação em nuvem é essencial.

Quando a computação em nuvem é contratada nos níveis empresariais, ela permite que as organizações foquem esforços na construção e transformação do conhecimento, deixando de investir em infraestrutura de rede e servidores de dados. Como estes são ativos caros, as pequenas e médias empresas podem usufruir dessa tecnologia, já que antigamente era praticamente inviável o investimento em sistemas de conhecimento e ERP (Enterprise Resources Planning).

\subsubsection{Big Data}

Diante desse cenário de tudo estar conectado e alimentando a nuvem, estima-se que até 2020, 1,7 Megabytes de dados serão gerados a cada segundo (MARR, 2015), o que forma o Big Data. Esses dados precisam ser minerados e tratados, para que a informação necessária seja transformada em conhecimento com valor agregado.

Há técnicas de mineração de dados com auxílio de Inteligência Artificial (IA) que permitem a extração do conhecimento da imensidão de dados da nuvem. Adicionalmente, como a microeletrônica permitiu a inserção de inteligência em pequenos dispositivos eletrônicos, através de microprocessadores cada vez menores, a informação desejada já pode ser captada diretamente no nível de sensores e enviada a nuvem. Como esse processo está em um nível "atmosférico" baixo, ou seja, no chão-de-fábrica ou a poucos metros do nível do "solo", a técnica é chamada de Fog Computing (CISCO SYSTEMS, 2016) - “Computação na Neblina”.

\subsubsection{Integração Vertical e Horizontal de Sistemas}

Se as pessoas precisam estar conectadas e as empresas também, há a preocupação de integração de sistemas e a interoperabilidade deles. A integração de sistemas é um conceito 
presente desde a $3^{\text {a }}$ Revolução Industrial, mas era feita apenas de forma vertical e somente as grandes empresas conseguiam possuir, devido aos altos custos dos próprios sistemas e técnicas de integração. Além disso, os desenvolvedores de sistemas de automação optaram por proteger suas marcas empregando protocolos de comunicação proprietários, fazendo com que as organizações se tornassem seus reféns.

Com a TD, o objetivo é que, independentemente de marcas, os sistemas sejam integrados facilmente, desde o chão-de-fábrica até os níveis gerenciais. Também de forma horizontal (M2M), mas além das fronteiras das organizações, conectando toda a cadeia de suprimentos e logística. A Gestão do Conhecimento torna-se uma ferramenta essencial para que essa integração seja possível.

\subsubsection{Segurança Cibernética}

Se os sensores de mais baixo nível são conectados à Internet, na nuvem e em diversos outros sistemas, eles podem estar sujeitos aos ciber-ataques. Dessa maneira, é possível dizer que o conhecimento das empresas também está sob risco. Nesse sentido, há de se pensar em defesa em profundidade, que exige investimentos em arquiteturas de rede confiáveis, com configuração segura de dispositivos e gestão de segurança em redes (WOOD, 2017). Garantir a segurança cibernética exige gestão de conhecimento específico na área de segurança de sistemas de informação.

\subsubsection{Manufatura Aditiva}

Impressoras 3D tem se popularizado para mercados amadores, mas também em projetos de engenharia e já em processos produtivos. A impressão 3D tem permitido a redução significativa de custos e tempo com a construção de protótipos. Com o avanço da tecnologia, alguns processos produtivos já têm usado as impressoras para a produção de peças finais, como a Boeing, que imprime peças em titânio, através de sinterização a laser. É uma disrupção na manufatura, que tradicionalmente tem usado as máquinas $\mathrm{CNC}$.

\subsubsection{Simulação}

A diminuição de custos com protótipos não vem apenas da impressão 3D, mas também de Simulação Computacional. Ela já era possível com softwares do tipo CAD/CAM e para a 
validação de processos produtivos. Mas com o avanço da tecnologia empregada em computadores, a Simulação também evoluiu. Computadores com hardware robusto e softwares mais avançados permitem que fábricas inteiras sejam simuladas, inclusive com logística interna e externa. E com visão tridimensional! O ganho de tempo é uma grande vantagem desse pilar. Ainda mais se for aliada a Realidade Aumentada.

\subsubsection{Realidade Aumentada}

Muitas organizações têm usado a tecnologia para a visualização de produtos e serviços ainda não produzidos. Os protótipos são simulados com o uso da Realidade Aumentada, para validação virtual. Mas além disso, a tecnologia já tem sido usada como Realidade Mista em processos produtivos e serviços. Com o uso dos smartglasses e tablets ou smartphones, é possível se ter informações da produção em tempo real com ou sem imersão, respectivamente. Para algumas situações, através de gestos com as mãos, é possível ter interação com o ambiente virtual e operar o ambiente real. Olhando dessa forma, é correto dizer que a Realidade Aumentada ou Mista, aliadas a IoT e Integração de Sistemas, habilita os CPS, que, consequentemente, tem muito a colaborar com a Gestão do Conhecimento.

\subsection{GESTÃO DO CONHECIMENTO}

O conhecimento é um ativo valioso para as organizações que está em constante atualização. Isso se deve ao fato de que o conhecimento está inserido em um ciclo de gestão (Figura 3), que parte da construção do conhecimento e depois é transformado. Se passou por um processo de transformação, o conhecimento precisa ser organizado, para então ser implementado e em seguida, usado (WIIG, 1997).

A criação do conhecimento organizacional é um processo que torna disponível e amplifica o conhecimento criado individualmente, bem como converte e o conecta ao sistema de conhecimento da empresa (NONAKA; VON KROGH; VOELPEL, 2006). Isso é explicado pela teoria de criação do conhecimento organizacional, que tem sido utilizada para explicar, por exemplo, a gestão de tecnologia e sistemas de gestão da informação.

A Gestão do Conhecimento (GC) gera valor e cria conhecimentos novos, tornando as empresas competitivas. Mas competitividade requer a criação e a manutenção do portfólio do capital intelectual, para se fortalecer nas áreas que definem os negócios empresariais. E tem sido assim que o papel do conhecimento, em aumentar a competitividade, mostrou como a transformação e aplicação do conhecimento na geração de valor é essencial para o sucesso 
empresarial (WIIG, 1997). No contexto da TD, o uso da tecnologia da informação e da IA como suporte para a GC, permitem a extração das informações que podem ser transformadas em conhecimento. Dessa forma, a Engenharia do Conhecimento, se favorecendo das técnicas de IA, como machine learning, faz com que as melhores práticas dos trabalhadores sejam aprendidas, codificadas e armazenadas como conhecimento (LYDON, 2016).

Figura 3. Ciclo do Conhecimento

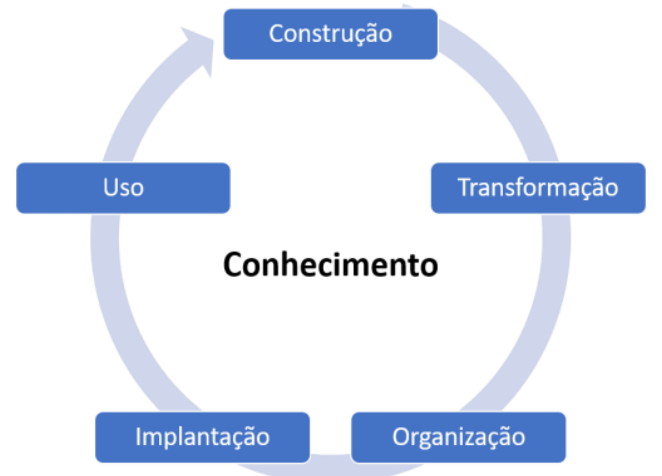

Fonte: (WIIG, 1997).

Contudo, a identificação, captura e transferência do conhecimento devem ser consonantes com os objetivos estratégicos organizacionais (MALONE, 2002). A tecnologia conduz as organizações a mirarem no que é explicito (mecanicista). Entretanto, para ser efetivo no atingimento dos objetivos, a GC tem que converter o conhecimento tácito da força de trabalho para explícito, ou seja, capturar e arquivar o conhecimento.

As empresas inovadoras e competitivas, que adotaram a GC, usam o conhecimento tácito formalizado para ter eficiência e eficácia nos processos administrativos, pois a formalização significa ter memória organizacional, tornando as tarefas fáceis e ágeis (LOTTI OLIVA, 2014).

E nessa perspectiva ampla da GC aliada a TD, as organizações se colocam em uma posição competitiva. Com objetivos comuns, essa união permite que os processos produtivos e os serviços oferecidos sejam competitivos, garantindo a permanência e consolidação dos negócios. As empresas que não mergulharem na onda da $4^{\mathrm{a}}$ Revolução Industrial assumem o risco da não-digitalização, fenômeno correlato das décadas de 80 até os anos 2000, que era o risco da não-automatização (GROOVER, 2011). 


\section{PROCEDIMENTOS METODOLÓGICOS}

A pesquisa foi descritiva e exploratória, para tentar encontrar as contribuições da Gestão do Conhecimento na implementação da Transformação Digital nos negócios das organizações. Esta seção apresenta o design da pesquisa e termos adotados para encontrar os resultados.

\subsection{DESIGN DA PESQUISA}

Para responder à questão de pesquisa deste estudo, a base de dados Scopus foi definida como principal fonte de publicações. Além disso, também ficou definido inicialmente que apenas as 20 publicações mais citadas seriam usadas para este estudo.

A pesquisa na base de dados Scopus foi feita usando a combinação de "Industry 4.0" e “Knowledge Management", o que resultou em 55 trabalhos encontrados, mas apenas onze citados (ANSARI; SEIDENBERG, 2016; BURZLAFF; BARTELT, 2017; DOSSOU; NACHIDI, 2017; KAMP; OCHOA; DIAZ, 2017; MENON; KÄRKKÄINEN; GUPTA, 2016; POSSELT et al., 2016; SAMARANAYAKE; RAMANATHAN; LAOSIRIHONGTHONG, 2018; SCHEUERMANN et al., 2017; SHAMIM et al., 2017; STOCKER et al., 2014; SYNNES; WELO, 2016). Devido a esse baixo número, outras 9 publicações com alta citação e relevância para este estudo foram incorporadas, que acabou resultando em 20 publicações a serem analisadas (GRONAU; GRUM, 2017; KOTYNKOVA, 2017; LU, 2017; PFEIFFER, 2017; PFEIFFER; SUPHAN, 2015; PRIFTI et al., 2017; SHAFIQ et al., 2015; SONNTAG et al., 2017; VLADOVA; ULLRICH; SULTANOW, 2017).

Dessa forma, não foram aplicados critérios de exclusão, estratégias de pesquisa e elegibilidade de artigos. Os resultados foram sintetizados na próxima sessão, que servem de base para a discussão de resultados.

\subsection{TERMOS ADOTADOS}

A Transformação Digital possui sinônimos, um deles, já citado aqui diversas vezes, é a Indústria 4.0. Outros termos também são usados frequentemente, como Fábricas Inteligentes, Empresa Conectada, Digitalização e $4^{\mathrm{a}}$ Revolução Industrial. Quando esses termos foram encontrados nas publicações, eles foram considerados como sinônimos de Transformação Digital e Indústria 4.0.

Para relacionar a Transformação Digital com a Gestão do Conhecimento, os nove pilares também são considerados. Como trata-se de tema extremamente amplo, as publicações não 
abordam a Indústria 4.0 como um todo, mas sim um ou mais pilares em suas pesquisas. Dessa forma, os sinônimos para os pilares também são levados em conta nas leituras.

Além dos nove pilares, temas relacionados a sistemas ciber-físicos (CPS) são considerados, pois CPS são habilitadores da Indústria 4.0.

\section{RESULTADOS}

Nessa sessão, uma breve análise será realizada para cada publicação encontrada, relacionando a contribuição da Gestão do Conhecimento para a implementação da Transformação Digital, e vice-versa. Para tanto, a Tabela 1 contém as relações da GC com os pilares da TD e, também, com os Sistemas Ciber-Físicos (CPS). Um "X” (caixa alta) apresenta a principal contribuição da publicação, enquanto que um " $x$ " (caixa baixa) significa que ela cita algum pilar, mas não faz parte da principal contribuição. Portanto, um "X" é um relacionamento único da publicação com algum pilar e o " $x$ ” pode apresentar mais de um cruzamento. Algumas publicações marcadas com “*” (KOTYNKOVA, 2017; LU, 2017; PFEIFFER, 2017; PFEIFFER; SUPHAN, 2015; PRIFTI et al., 2017; VLADOVA; ULLRICH; SULTANOW, 2017) não apresentam um pilar como foco de sua contribuição, nesse caso, os autores abordaram vários pilares da Indústria 4.0, mas não todos.

A coluna "Contribuição" da Tabela 1 diz o sentido da colaboração de cada trabalho, na relação entre GC com TD.

\subsection{CPS E GC}

A principal contribuição de SHAFIQ et al. (2015) foi a definição formal de um mecanismo ou artefato de engenharia (VEO - Virtual Engineering Object) que trata da implementação da Engenharia do Conhecimento no campo da manufatura para CPS para tomada de decisão. O VEO é uma representação do conhecimento de objetos de engenharia que agrega conhecimento e experiência. Também tem a capacidade para adicionar, armazenar, melhorar e compartilhar conhecimento baseado em experiência. O VEO é uma especialização dos CPS, em termos de sua extensão dentro da coleta e reuso do conhecimento. Seu conceito usa uma técnica de representação do conhecimento chamada SOEKS (Conjunto de Estruturas do Conhecimento e Experiências para gerenciamento de conhecimento explícito), para tomada de decisão nas empresas, como estrutura única e simples para captura, armazenamento, melhoramento e reuso de experiência decisória. Um VEO pode encapsular conhecimento e 
experiência de cada característica importante relacionada com o objeto de engenharia, como conhecimento armazenado ou conhecimento operacional. Como o VEO é uma especialização dos CPS, verifica-se que o mecanismo é uma tecnologia no contexto da TD que contribui para a GC.

Tabela 1. Relacionamento das publicações com os pilares da Indústria 4.0 e CPS.

\begin{tabular}{|c|c|c|c|c|c|c|c|c|c|c|c|}
\hline Publicação & Contribuição & Robótica & $\begin{array}{l}\text { Integração } \\
\text { de sistemas }\end{array}$ & IoT & Simulação & $\begin{array}{l}\text { Manuf. } \\
\text { aditiva }\end{array}$ & $\begin{array}{c}\text { Computação } \\
\text { em nuvem }\end{array}$ & $\begin{array}{l}\mathbf{R} \\
\mathbf{A}\end{array}$ & $\begin{array}{c}\text { Big } \\
\text { Data } \\
\end{array}$ & $\begin{array}{l}\text { Segurança } \\
\text { cibernética }\end{array}$ & CPS \\
\hline (GRONAU; GRUM, 2017) & $\mathrm{TD} \rightarrow \mathrm{GC}$ & & & $\mathrm{x}$ & & & & $\mathrm{X}$ & & & $\mathrm{x}$ \\
\hline (SHAFIQ et al., 2015) & $\mathrm{TD} \rightarrow \mathrm{GC}$ & & & $\mathrm{x}$ & & & & & & & $\mathrm{X}$ \\
\hline $\begin{array}{c}\text { (KAMP; OCHOA; DIAZ, } \\
\text { 2017) }\end{array}$ & $\mathrm{TD} \rightarrow \mathrm{GC}$ & & $\mathrm{x}$ & $\mathrm{X}$ & & & $\mathrm{x}$ & & $\mathrm{x}$ & $\mathrm{x}$ & $\mathrm{x}$ \\
\hline $\begin{array}{c}\text { (MENON; } \\
\text { KÄRKKÄINEN; GUPTA, } \\
\text { 2016) }\end{array}$ & $\mathrm{TD} \rightarrow \mathrm{GC}$ & & $\mathrm{x}$ & $\mathrm{X}$ & & & $\mathrm{x}$ & & $\mathrm{x}$ & & $\mathrm{x}$ \\
\hline $\begin{array}{c}\text { (DOSSOU; NACHIDI, } \\
\text { 2017) }\end{array}$ & $\mathrm{TD} \leftrightarrow \mathrm{GC}$ & $\mathrm{x}$ & $\mathrm{x}$ & $\mathrm{x}$ & $\mathrm{X}$ & & & & & & \\
\hline (STOCKER et al., 2014) & $\mathrm{TD} \rightarrow \mathrm{GC}$ & & & & & & & $\mathrm{X}$ & & & \\
\hline $\begin{array}{c}\text { (BURZLAFF; BARTELT, } \\
\text { 2017) }\end{array}$ & $\mathrm{TD} \leftarrow \mathrm{GC}$ & & $\mathrm{X}$ & $\mathrm{x}$ & & & & & & & \\
\hline (POSSELT et al., 2016) & $\mathrm{TD} \rightarrow \mathrm{GC}$ & & & & & $\mathrm{x}$ & & & & & $\mathrm{X}$ \\
\hline $\begin{array}{c}\text { (SCHEUERMANN et al., } \\
\text { 2017) }\end{array}$ & $\mathrm{TD} \rightarrow \mathrm{GC}$ & & & $\mathrm{x}$ & & & & & & & $\mathrm{X}$ \\
\hline (SHAMIM et al., 2017) & $\mathrm{TD} \leftarrow \mathrm{GC}$ & & & $\mathrm{x}$ & & & & & & & $\mathrm{X}$ \\
\hline (SYNNES; WELO, 2016) & $\mathrm{TD} \leftarrow \mathrm{GC}$ & $\mathrm{x}$ & $\mathrm{X}$ & & $\mathrm{x}$ & $\mathrm{x}$ & & $\mathrm{x}$ & & & \\
\hline $\begin{array}{c}\text { (ANSARI; SEIDENBERG, } \\
\text { 2016) }\end{array}$ & $\mathrm{TD} \leftarrow \mathrm{GC}$ & $\mathrm{x}$ & $\mathrm{x}$ & & & $\mathrm{x}$ & & $\mathrm{x}$ & & & $\mathrm{X}$ \\
\hline $\begin{array}{c}\text { (SAMARANAYAKE; } \\
\text { RAMANATHAN; } \\
\text { LAOSIRIHONGTHONG, } \\
2018 \text { ) }\end{array}$ & $\mathrm{TD} \leftrightarrow \mathrm{GC}$ & & $\mathrm{X}$ & & & & & & & & \\
\hline (PRIFTI et al., 2017)* & $\mathrm{TD} \leftarrow \mathrm{GC}$ & $\mathrm{x}$ & $\mathrm{x}$ & $\mathrm{x}$ & & & $\mathrm{x}$ & & $\mathrm{x}$ & $\mathrm{x}$ & \\
\hline $\begin{array}{l}\text { (VLADOVA; ULLRICH; } \\
\text { SULTANOW, 2017)* }\end{array}$ & $\mathrm{TD} \leftarrow \mathrm{GC}$ & & $\mathrm{x}$ & $\mathrm{x}$ & & & & & & & \\
\hline$(\mathrm{LU}, 2017) *$ & $\mathrm{TD} \leftrightarrow \mathrm{GC}$ & & $\mathrm{x}$ & $\mathrm{x}$ & & & $\mathrm{x}$ & $\mathrm{x}$ & $\mathrm{x}$ & $\mathrm{x}$ & $\mathrm{x}$ \\
\hline (SONNTAG et al., 2017) & $\mathrm{TD} \rightarrow \mathrm{GC}$ & $\mathrm{x}$ & $\mathrm{x}$ & $\mathrm{x}$ & & & $\mathrm{x}$ & $\mathrm{x}$ & $\mathrm{x}$ & & $\mathrm{X}$ \\
\hline (KOTYNKOVA, 2017)* & Não tem & $\mathrm{x}$ & & $\mathrm{x}$ & & & & & $\mathrm{x}$ & & $\mathrm{x}$ \\
\hline $\begin{array}{c}\text { (PFEIFFER; SUPHAN, } \\
2015)^{*}\end{array}$ & $\mathrm{TD} \leftarrow \mathrm{GC}$ & $\mathrm{x}$ & $\mathrm{x}$ & $\mathrm{x}$ & & $\mathrm{x}$ & & $\mathrm{x}$ & $\mathrm{x}$ & $\mathrm{x}$ & $\mathrm{x}$ \\
\hline (PFEIFFER, 2017)* & $\mathrm{TD} \leftrightarrow \mathrm{GC}$ & $\mathrm{x}$ & $\mathrm{x}$ & $\mathrm{x}$ & $\mathrm{x}$ & $\mathrm{x}$ & & & $\mathrm{x}$ & $\mathrm{x}$ & $\mathrm{x}$ \\
\hline Total de relações & $\begin{array}{c}\mathrm{TD} \rightarrow \mathrm{GC}=8 \\
\mathrm{TD} \leftarrow \mathrm{GC}=7 \\
\mathrm{TD} \leftrightarrow \mathrm{GC}=4 \\
\text { não tem }=1\end{array}$ & 8 & 13 & 15 & 3 & 5 & 5 & 7 & 8 & 5 & 14 \\
\hline $\begin{array}{c}\text { Total de relações } \\
\text { principais }\end{array}$ & & 0 & 3 & 2 & 1 & 0 & 0 & 2 & 0 & 0 & 6 \\
\hline
\end{tabular}

Apesar de não focar em CPS, há mais um trabalho relacionado ao conhecimento explícito, que é baseado em experiência e que avaliou a capacidade e experiência de pessoal e recursos para a Industria 4.0 (PFEIFFER; SUPHAN, 2015), permitindo dizer que a GC tem contribuído para a implementação da TD. A implementação bem-sucedida dependerá decisivamente da capacidade de gerar e usar conhecimento baseado em experiência, o que será indispensável para a organização da Indústria 4.0 e para seu bom funcionamento.

A publicação (PFEIFFER; SUPHAN, 2015) relata que o conhecimento baseado na experiência do funcionário assume uma importância maior em tarefas não rotineiras, pois as tarefas repetitivas de produção se tornam cada vez mais digitalizadas. Na verdade, o conhecimento dinâmico, baseado na experiência, mostrou desempenhar um papel importante, especialmente naqueles trabalhos e tarefas que são comumente associados às economias de 
serviço e conhecimento, que incluem, por exemplo, a gestão de conhecimento e gestão de projetos.

Outras 5 publicações relacionadas a GC estão focadas em CPS (ANSARI; SEIDENBERG, 2016; POSSELT et al., 2016; SCHEUERMANN et al., 2017; SHAMIM et al., 2017; SONNTAG et al., 2017). O primeiro (ANSARI; SEIDENBERG, 2016) fala da colaboração entre humanos e CPS na resolução de problemas. Como há introdução de novas tecnologias, deve-se investir em habilidades das pessoas, conhecimento e aprendizagem organizacional em uma perspectiva estratégica, pois avanços na tecnologia frequentemente requerem mudanças na organização para se alcançar ganhos de produtividade. Isso incluiu investimentos em termos de capital e aquisição do conhecimento. Portanto, nessa publicação, conclui-se que a GC é um meio para a TD.

O segundo trabalho relacionado a CPS, aborda um sistema de gerenciamento de aprendizagem (LMS) inteligente com sensores em malha fechada (POSSELT et al., 2016). A ideia é que o aprendiz adquira o conhecimento através de uma fábrica de aprendizagem, que pode ser real ou virtual, como bancadas didáticas, conectadas ao LMS. Também aborda a interatividade para transferir o conhecimento através de uma abordagem multissensorial em processos de malha fechada. Os conhecimentos específicos podem ser treinados em fábricas de aprendizagem, que podem ser transferidos e, também, adquiridos. O sistema como um todo promove a motivação e a satisfação do aprendiz, melhorando o conhecimento experimental. Além disso, o computador adapta a velocidade de aprendizagem e possibilita a avaliação do crescimento do conhecimento através de testes. Para o objetivo de pesquisa deste trabalho, verificou-se que, nesse caso, foi a TD que contribuiu para a GC.

Mais uma contribuição da TD para a GC está presente no terceiro trabalho, que propõe um CPS, com uso de wearables para monitoração de incidentes e análise na manutenção industrial e compartilhamento do conhecimento adquirido ou gerado de forma horizontal, nos mesmos níveis industriais, mas além das fronteiras da organização e de forma vertical na empresa e além dela. Assim, dados de produtividade de equipamentos poderiam ser compartilhados, ou seja, conhecimento (SCHEUERMANN et al., 2017). Isso porque um objetivo da Indústria 4.0 é melhorar o compartilhamento horizontal e vertical entre empresas. A horizontal para compartilhar conhecimento entre áreas e vertical entre a hierarquias. Sendo que na primeira é feito o compartilhamento do conhecimento, especialmente sobre incidentes de manutenção, resultando em produtos melhores, menores tempos de parada e melhoria a eficiência da indústria. Já o compartilhamento vertical é sobre o conhecimento de vendas, pósvendas, estoques, manutenção preventiva, compras, setup, qualidade e outros. 
A quarta contribuição relacionada a CPS debate a viabilidade da Indústria 4.0 para o setor hospitaleiro (setor de serviços) sobre as lentes de práticas de gestão, pois são requeridas inovação e aprendizagem contínua (SHAMIM et al., 2017). Como há alta rotatividade de funcionários nos hotéis, o conhecimento tácito é perdido facilmente. Então, há a necessidade de converter o conhecimento tácito dos empregados em conhecimento organizacional. Para tanto, é preciso alinhar as práticas da GC, como a premiação dos empregados por contribuições, a performance na aprendizagem e a proposta de inovação, com a aceitação da tecnologia na economia baseada no conhecimento. Os treinamentos precisam ser planejados para levar a implementação de CPS, ou seja, a GC está contribuindo para a implementação da Indústria 4.0.

Já o último trabalho trata da visão geral de CPS para projeto de fábricas inteligentes, com aquisição do conhecimento (SONNTAG et al., 2017). Os autores descrevem uma infraestrutura para aquisição do conhecimento, incluindo o uso de IoT, que inclui caso de uso para a GC.

\subsection{RA E GC}

Há dois trabalhos que usam a Realidade Aumentada (RA) de maneira a contribuir com a GC (GRONAU; GRUM, 2017; STOCKER et al., 2014). De novo, contribuições no sentido da TD colaborando para a GC.

No primeiro trabalho (GRONAU; GRUM, 2017), métodos de modelagem de bases do conhecimento tácito e habilidades para representar o conhecimento são usados, além de permitir a transferência simples ou numerosa do conhecimento. A RA coloca ao operador entidades do conhecimento, que ele visualiza de forma transparente junto a máquina real. Esse cenário faz com que haja diferentes visões para a conversão do conhecimento, consequentemente, diversas modelagens. Na criação de cenários, um dos objetivos é que o mundo real para transferência do conhecimento tácito tem que ser visualizado. Conclui-se que um ambiente de aplicação da Indústria 4.0, onde há atividades intensivas em conhecimento, é um cenário propício para a transferência do conhecimento tácito, simulação de processos e coordenação de esforços.

Na segunda contribuição (STOCKER et al., 2014b), óculos de RA são aplicados para a gravação de vídeos das tarefas de um trabalhador experiente. Assim, o trabalhador menos experiente pode recuperar as mídias para adquirir o conhecimento. Dessa forma, a GC poderia ser facilmente aplicada com gestos e toques, com o uso de tecnologias centradas no ser humano em fábricas inteligentes. 


\subsection{INTEGRAÇÃO DE SISTEMAS E GC}

Foram encontradas três publicações relacionadas a Integração de Sistemas, como principal contribuição (BURZLAFF; BARTELT, 2017; SAMARANAYAKE; RAMANATHAN; LAOSIRIHONGTHONG, 2018; SYNNES; WELO, 2016).

Os primeiros autores abordam a composição de arquitetura dirigida ao conhecimento que permite reuso da integração do conhecimento (BURZLAFF; BARTELT, 2017). Há uma crítica na publicação dizendo que as arquiteturas dinâmicas para IIoT não tem sido usadas e que predominam especificações informais, o que impede o reuso do conhecimento. Assim é proposto que as especificações de interface devem ser feitas com técnicas de integração especificas com as técnicas de GC. A proposta é de uma base do conhecimento estendida passoa-passo com a integração semântica formal do conhecimento resultante de uma tarefa manual específica. A combinação de várias dessas atividades serve como uma base para reuso do conhecimento. Nessa publicação, pode-se concluir que é a GC que apoia a TD.

A segunda contribuição para Integração de Sistemas é uma revisão sistemática sobre implementação da Indústria 4.0 (SAMARANAYAKE; RAMANATHAN; LAOSIRIHONGTHONG, 2018). Os autores citam que a conectividade e integração são conceitos amplos e chaves para as diversas áreas, que incluiu a integração de sistemas ERP e MES com Sistemas de Gestão do Conhecimento (KMS) e BPMS. Dessa forma, conclui-se que a colaboração entre GC e TD tem via de mão-dupla.

E a última publicação sobre Integração de Sistemas fala em como aumentar a capacidade de integração da produção enxuta com o desenvolvimento de processo, usando o contexto da Indústria 4.0 (SYNNES; WELO, 2016). Os autores relatam que nesse paradigma, as empresas precisam aumentar a capacidade de integração e facilitar o compartilhamento do conhecimento entre a engenharia do produto e a produção para obter vantagem competitiva. Ainda, há a necessidade de investir em habilidade de pessoas, conhecimento e aprendizagem organizacional, como perspectiva estratégica. Isto porque avanços na tecnologia requerem mudanças na organização para aumento da produtividade, o que inclui investimentos em capital e aquisição do conhecimento. Os autores fornecem o exemplo da Rolls-Royce, onde o conhecimento em automação foi alavancado pela combinação de especialistas externos com especialistas internos. Isto garantiu uma equipe multidisciplinar que possui conhecimento em tecnologias para desenvolver uma solução automatizada para a produção do produto. Considerando o contexto da GC, é ela que faz a TD acontecer. 


\subsection{IOT E GC}

Para o pilar da IoT, apesar da sua importância na Transformação Digital, apenas dois trabalhos focam em soluções para a GC (KAMP; OCHOA; DIAZ, 2017; MENON; KÄRKKÄINEN; GUPTA, 2016), portanto, a TD nesse caso é o meio para a GC.

O primeiro trata da "servitização", que é a união da venda do produto com a venda de serviços embutidos. (KAMP; OCHOA; DIAZ, 2017). O exemplo da publicação é a relação entre o fornecedor de máquinas-ferramenta e clientes da indústria de manufatura, com interação através de modelos de conhecimento intensivo. A ideia é tornar as máquinas-ferramentas dispositivos com "IoT", ou seja, conectadas e inteligentes. Dessa forma, o fabricante da máquina poderá obter conhecimento de como os clientes usam as máquinas, para assim, poder oferecer serviços sobre esse conhecimento adquirido e criar modelos de negócios, como o pagamento por uso e não por aquisição do equipamento (gastos com operação contra gastos com investimentos).

O segundo trabalho aborda a Internet Industrial para o gerenciamento de vida de produto (PLM) relacionando informação e conhecimento (MENON; KÄRKKÄINEN; GUPTA, 2016). A Internet Industrial tem o papel de melhorar a GC relacionado ao ciclo de vida do produto em diferentes fases, pois ela provê novos meios para acessar e acelerar a captura de dados e convertê-los conhecimento perspicaz. Os autores citam os desafios da GC e do PML, pois ambos têm objetivos semelhantes e criam valor ao cliente. Um PLM usa uma plataforma de compartilhamento para capturar, representar, organizar, recuperar e reusar informação sobre ciclo de vida de produtos nas organizações e suporta a integração com outros softwares. Os desafios da GC no contexto do PLM estão relacionados ao conhecimento estruturado, nãoestruturado e tácito dos empregados.

\subsection{SIMULAÇÃO E GC}

Um único trabalho relacionado a GC aborda a Simulação como suporte para modelar a cadeia de suprimentos (DOSSOU; NACHIDI, 2017). O objetivo foi fornecer e testar cenários em um sistema de simulação para medição dos impactos das mudanças e decisões estudados para o sistema de supply chain, com a aplicação de veículos guiados automatizados (AGV). O uso da simulação tem facilitado a determinação das soluções mais adequadas para as empresas. A ideia de combinar melhorias, modelagem e simulação foi validada na aplicação dos autores. Entende-se que nessa proposta, tanto a GC como a TD têm colaboração entre si. 


\subsection{GC E OS OUTROS PILARES DA GC}

Dois trabalhos falam sobre competências para a Indústria 4.0. O primeiro propõe um modelo de competências para os empregados da Indústria 4.0 (PRIFTI et al., 2017). O modelo sugerido coloca a GC na Criação e Conceitualização, sendo que estas são grandes competências para o trabalhador da Indústria 4.0. Contudo, o estudo dos autores mostra que a maioria das competências requeridas são comportamentais e apenas uma pequena parte está relacionada ao conhecimento de domínio. As vagas de emprego na economia atual geralmente se concentram em uma lista de conhecimentos de domínio e incluem apenas algumas competências comportamentais muito genéricas, como o trabalho em equipe ou o trabalho independente. Esses exemplos mostram que o conhecimento do domínio é o foco da economia atual, enquanto que a Indústria 4.0 mudará o ambiente de trabalho. A TD conduzirá os trabalhadores a ambientes de trabalho dinâmicos, internacionais e interdisciplinares, portanto, competências como trabalho em ambientes interdisciplinares, flexibilidade, adaptabilidade, inovação, criatividade, pensamento crítico e gerenciamento de mudanças ganham uma nova importância. Por serem capazes de sempre se adaptar as mais recentes tecnologias e tirar o máximo proveito delas, os profissionais devem aplicar a aprendizagem ao longo da vida e a gestão do conhecimento.

Outro ponto é que os profissionais em TI deverão ter conhecimento em segurança cibernética, dados e redes, bem como integração de sistemas, já que estarão em trabalho constante com engenheiros. Também precisam saber sobre sistemas embarcados e sensores, robótica e IA, computação em nuvem e big data. Essa dimensão de competências representa o conhecimento de domínio, portanto, dependendo do domínio, os funcionários devem trazer diferentes competências (PRIFTI et al., 2017).

A segunda contribuição (VLADOVA; ULLRICH; SULTANOW, 2017) sugere o desenvolvimento de competências orientadas a demanda no contexto da manufatura, com a relevância do processo e modelagem do conhecimento. A gestão de competências é um fator crucial de sucesso para as organizações na interseção da gestão do conhecimento, da gestão de recursos humanos e da gestão de processos. Dessa maneira, deve ser considerada a partir de uma perspectiva da economia do conhecimento. A economia do conhecimento é um campo de crescente interesse na área de gestão do conhecimento e lida com o impacto financeiro da criação, modificação, distribuição e uso do conhecimento.

No contexto da Indústria 4.0, os autores apresentam um modelo onde a GC identifica os recursos do conhecimento, instruções de trabalho, documentos, etc. para a gestão de 
competências. Enquanto que essa identifica as necessidades de conhecimento para a GC e gerencia o capital intelectual. Por outro lado, a gestão de recursos humanos e a gestão do conhecimento fornecem artefatos para melhorar as competências. Dessa forma, a gestão propositada de competências e de pessoal afeta diferentes domínios organizacionais e conectam o gerenciamento de recursos humanos e do conhecimento com os objetivos estratégicos gerais da empresa. O desenvolvimento dos recursos de conhecimento ao longo do tempo permite que a empresa adquira e sustente vantagens competitivas.

LU (2017) aponta que os objetivos da Indústria 4.0 são altos índices de eficiência e produtividade operacional, bem como altos níveis de automatização. Otimização e customização da produção, automação e adaptação, agregação de valor nos serviços e negócios, troca automática de dados e comunicações são os maiores recursos da Indústria 4.0. Isso indica que é um processo industrial de adição de valor e gestão do conhecimento.

Contudo, o autor identifica que a IoT transfere e integra conhecimento entre organizações e setores das organizações. Portanto, ela melhora a eficiência e efetividade da GC na Indústria 4.0, sendo uma nova geração de economia baseada em conhecimento.

A transformação do trabalho produtivo na visão da Indústria 4.0 sobre a fabricação é tratada por PFEIFFER (2017) e criticada por KOTYNKOVA (2017). Algoritmos inteligentes e Big Data podem substituir trabalhadores de conhecimento qualificados, pelo menos parcialmente. Da mesma forma, robôs provavelmente serão usados em ambientes de produção que até agora resistiram à automação. As empresas não podem se dar ao luxo de meramente obter conhecimento, mas precisam se adaptar a uma estratégia de aquisição permanente de conhecimento. Agora, há uma oportunidade de aprender mais rápido e impulsionar melhorias de desempenho, aproveitando a transmissão de conhecimento. Nesse ambiente, a GC e TD colaboram entre si.

\subsection{ANÁLISE DOS RESULTADOS}

Um único trabalho não apresenta a integração da GC com a TD (KOTYNKOVA, 2017). O foco é uma análise crítica da Indústria 4.0 e como ela pode afetar o mercado de trabalho, evidenciando que os trabalhadores do conhecimento estão sendo reduzidos pela introdução das tecnologias que essa nova indústria traz consigo.

Contudo, olhando novamente para a Tabela 1, especificamente na coluna "Contribuições", conclui-se que a Transformação Digital é meio para que a Gestão do Conhecimento melhore ainda mais os negócios empresariais. Isso foi visto em 8 publicações. 
Com certeza, a era da Indústria 4.0 possui tecnologias que favorecem esse cenário, embarcando tecnologias na GC, como os CPS, a IoT e a Realidade Aumentada.

Já em 7 trabalhos, verificou-se o contrário: é a Gestão do Conhecimento que contribui para a Transformação Digital das organizações, seja em seus produtos, procedimentos organizacionais ou processos produtivos. No pilar da Integração de Sistemas Horizontais e Verticais, esse tipo de colaboração fica evidente, mas também em alguns casos de uso de CPS. A Integração de Sistemas exige conhecimento especifico de diversas áreas, bem como a implementação de CPS. Portanto, o conhecimento para a implementação da TD precisa ser gerenciado.

Mas é importante dizer que a colaboração mútua entre GC e TD foi encontrada em 4 publicações. No pilar da Simulação, essa evidência aparece, pois é uma tecnologia que precisa da GC para a construção do ambiente virtual e o contrário fornece conhecimento sobre o que está sendo simulado. As tecnologias que contem IoT, CPS e Realidade Aumentada também favorecem esse caminho de mão-dupla.

Independentemente do sentido da colaboração entre GC e TD, pode-se dizer que o ciclo do conhecimento, da Figura 3, é constantemente utilizado. Todo o conhecimento que vem sendo construído ao longo dessa última Revolução Industrial, passou por um processo de transformação, organização, implantação e uso. Por exemplo, o sensor de contagem de peças metálicas em uma esteira. A sua construção usa o conhecimento sobre eletromagnetismo, materiais e eletrônica para a detecção de peças. Toda vez que há a sensibilização do dispositivo, o dado eletrônico é enviado a um controlador que faz a contagem. Com a transformação, ou evolução da microeletrônica, os conhecimentos precisaram ser organizados para a implantação de processamento e comunicação ao sensor. Agora ele é usado para, além de contar peças, informar diretamente ao usuário final outros dados, como o tempo entre a contagem de uma peça e outra, valores de corrente e tensão elétricas e situações de falhas.

\section{CONSIDERAÇÕES FINAIS}

A Transformação Digital (TD) tem modificado a forma como os processos organizacionais são implementados e gerenciados com a inclusão de novas tecnologias, como Realidade Aumentada, IoT e Sistemas Ciber-Fïsicos (CPS), para promover Gestão do Conhecimento (GC). Mas é possível dizer que a GC também é meio para a TD ser implementada, pois a Integração de Sistemas e os CPS contribuem para esse tipo de relação. Todavia, a GC e TD colaborando entre si ao mesmo tempo é uma realidade, quando se vê que 
os CPS, Integração de Sistemas e Simulação são tecnologias capazes de promover o relacionamento em via dupla. Portanto, as técnicas usadas pela GC, aliadas à Indústria 4.0, podem ser melhor aplicadas em busca da melhoria dos processos e da memória organizacional.

Publicações enfatizando a Manufatura Aditiva, a Robótica, a Computação em Nuvem, a Segurança Cibernética ou o Big Data não foram encontradas. O conhecimento existente nos três primeiros pilares é vasto e precisa ser gerenciado, portanto, contribuições futuras nessas áreas poderiam ser realizadas. Para o Big Data, a mineração do dado que possa ser transformado em conhecimento tem relevância. Para esse pilar da TD, a Engenharia do Conhecimento, com suas técnicas de Inteligência Artificial, pode contribuir significativamente.

Mesmo que trabalhos tenham sido encontrados para a maioria dos pilares, constata-se que pouco tem se publicado a respeito do relacionamento entre a TD com a GC. Portanto, há muito o que evoluir para que a GC e a TD, de modo integrado, promovam a melhoria dos processos organizacionais e produtivos.

\section{REFERÊNCIAS}

ABII. Associação Brasileira de Internet Industrial. Disponível em: <https://www.abii.com.br/>.

ANSARI, F.; SEIDENBERG, U. A portfolio for optimal collaboration of human and cyber physical production systems in problem-solving. Proceedings of the 13th International Conference on Cognition and Exploratory Learning in the Digital Age, CELDA 2016. Anais...2016

BRASIL. Perspectivas de especialistas sobre a manufatura avançada no brasil - 2016. [s.l: s.n.].

BRASIL. Estratégia Brasileira para a Transformação Digital. [s.l: s.n.].

BRASIL. Agenda brasileira para a Indústria 4.0. Disponível em:

$<$ http://industria40.gov.br/>.

BURZLAFF, F.; BARTELT, C. Knowledge-driven architecture composition: Case-based formalization of integration knowledge to enable automated component coupling. Proceedings - 2017 IEEE International Conference on Software Architecture Workshops, ICSAW 2017: Side Track Proceedings, p. 108-111, 2017.

CISCO SYSTEMS. Fog Computing and the Internet of Things: Extend the Cloud to Where 
the Things Are. Www.Cisco.Com, p. 6, 2016.

DOSSOU, P. E.; NACHIDI, M. Modeling Supply Chain Performance. Procedia Manufacturing, v. 11, p. 838-845, 2017.

GRONAU, N.; GRUM, M. Integration of Augmented Reality Technologies in Process Modeling - The Augmentation of Real World Scenarios With the KMDL. Proceedings of the Seventh International Symposium on Business Modeling and Software Design, $\mathrm{n}$. January, p. 206-215, 2017.

GROOVER, M. P. Automação industrial e sistemas de manufatura/ Mikell P. Groover ; tradução Jorge Ritter, Luciana do Amaral Teixeira, Marcos Vieira ; revisão técnica José Hamilton Chaves Gorgulho Júnior, 2011. Disponível em:

$<$ http://search.ebscohost.com/login.aspx?direct=true\&db=cat05351a\&AN=bib.110601\&site= eds-live >

IVI. Strategic implementation framework of industrial value chain for connected industries. Tokyo: [s.n.]. Disponível em: <https://iv-i.org/wp/wpcontent/uploads/2018/04/IVRA-Next_en.pdf>.

KAMP, B.; OCHOA, A.; DIAZ, J. Smart servitization within the context of industrial usersupplier relationships: contingencies according to a machine tool manufacturer. International Journal on Interactive Design and Manufacturing, v. 11, n. 3, p. 651-663, 2017.

KOTYNKOVA, M. Re-Industrialization of Europe: Industry4.0 and the Future of Work. European Scientific Journal, v. 7881, n. April, p. 249-256, 2017.

LOTTI OLIVA, F. Knowledge management barriers, practices and maturity model. Journal of Knowledge Management, v. 18, n. 6, p. 1053-1074, 2014.

LU, Y. Industry 4.0: A survey on technologies, applications and open research issues. Journal of Industrial Information Integration, v. 6, p. 1-10, 2017.

LYDON, B. Industry 4.0: Intelligent and flexible production. InTech, 2016.

MALONE, D. Knowledge management. A model for organizational learning. International Journal of Accounting Information Systems, v. 3, n. 2, p. 111-123, 2002.

MARR, B. Big Data: 20 Mind-Boggling Facts Everyone Must Read. Forbes, 2015.

MENON, K.; KÄRKKÄINEN, H.; GUPTA, J. P. Role of Industrial Internet platforms in 
the management of product lifecycle related information and knowledge. IFIP International Conference on Product Lifecycle Management. Anais...Tampere: Springer, 2016Disponível em: <http://link.springer.com/10.1007/978-3-319-54660-5>

PFEIFFER, S. The Vision of "Industrie 4.0" in the Making - a Case of Future Told, Tamed, and Traded. NanoEthics, v. 11, n. 1, p. 107-121, 2017.

PFEIFFER, S.; SUPHAN, A. The Labouring Capacity Index: Living Labouring Capacity and Experience as Resources on the Road to Industry 4.0. Stuttgart: [s.n.].

POSSELT, G. et al. Intelligent Learning Management by Means of Multi-sensory Feedback. Procedia CIRP. Anais...2016

PRIFTI, L. et al. A Competency Model for "Industrie 4.0" Employees. 13th International Conference on Wirtschaftsinformatik, p. 46-60, 2017.

RÜBMANN, M. et al. Industry 4.0: World Economic ForumThe Boston Consulting Group. [s.l: s.n.]. Disponível em:

<https://www.bcgperspectives.com/Images/Industry_40_Future_of_Productivity_April_2015 _tcm80-185183.pdf>.

SAMARANAYAKE, P.; RAMANATHAN, K.; LAOSIRIHONGTHONG, T. Implementing industry 4.0 - A technological readiness perspective. IEEE International Conference on Industrial Engineering and Engineering Management, v. 2017-Decem, p. 529-533, 2018.

SCHEUERMANN, C. et al. Incident Localization and Assistance System: A case study of a Cyber-Physical Human System. 2015 IEEE/CIC International Conference on Communications in China - Workshops, CIC/ICCC 2015, p. 57-61, 2017.

SHAFIQ, S. I. et al. Virtual engineering object (VEO): Toward experience-based design and manufacturing for industry 4.0. Cybernetics and Systems, v. 46, p. 35-50, 2015.

SHAMIM, S. et al. Examining the feasibilities of Industry 4.0 for the hospitality sector with the lens of management practice. Energies, v. 10, n. 4, 2017.

SMART MANUFACTURING LEADERSHIP COALITION. SMLC Forum: Priorities, Infrastructure, and Collaboration for Implementation of Smart Manufacturing. Washington: [s.n.]. Disponível em: $\langle$ https://smartmanufacturingcoalition.org/sites/default/files/smlc_forum_report_vf_0.pdf $>$.

SONNTAG, D. et al. Overview of the CPS for Smart Factories Project: Deep Learning, Knowledge Acquisition, Anomaly Detection and Intelligent User Interfaces. p. 487-504, 
2017.

STOCKER, A. et al. Mensch-zentrierte IKT-Lösungen in einer Smart Factory.

Elektrotechnik und Informationstechnik, v. 131, n. 7, p. 207-211, 2014.

SYNNES, E. L.; WELO, T. Enhancing Integrative Capabilities through Lean Product and Process Development. Procedia CIRP. Anais...2016

VLADOVA, G.; ULLRICH, A.; SULTANOW, E. Demand-oriented Competency Development in a Manufacturing Context: The Relevance of Process and Knowledge Modeling. p. 4424-4433, 2017.

WIIG, K. M. Knowledge Management: An Introduction and Perspective. Journal of Knowledge Management, v. 1, n. 1, p. 6-14, 1997.

WOOD, R. Three keys to designing and configuring secure industrial networks. InTech, 2017.

WUEBBEKE, J. et al. Made in China 2025MERICS Papers on China. [s.l: s.n.]. Disponível em:

$<$ https://www.merics.org/fileadmin/user_upload/downloads/MPOC/MPOC_Made_in_China_ 2025/MPOC_No.2_MadeinChina_2025.pdf>. 